\title{
The interaction of conditioned taste aversions and schedule-induced polydipsia: Availability of alternative behaviors
}

\author{
ANTHONY L. RILEY, DAVID B. PEELE, and KATHY D. RICHARD \\ The American University, Washington, D.C. 20016 \\ and \\ PAUL J. KULKOSKY \\ Edward W. Bourne Behavioral Research Laboratory \\ Cornell Medical Center, White Plains, New York 10605
}

\begin{abstract}
Animals poisoned following exposure to saccharin subsequently avoided the schedule-induced consumption of saccharin. While this suppression was transient for subjects who had access only to the saccharin solution during the free-food presentations, recovery of schedule-induced saccharin consumption was significantly retarded for subjects who had concurrent access to saccharin and a running wheel. It has been suggested that the transient suppression of scheduleinduced polydipsia by conditioned taste aversions results from the pellet-induced tendency to drink within the schedule-induced polydipsia procedure. That access to the running wheel reduces schedule-induced polydipsia in general and prolongs the suppression of schedule-induced polydipsia by taste aversions supports this view.
\end{abstract}

If rats are poisoned following the schedule-induced consumption of saccharin, they display only a weak and transient suppression of schedule-induced drinking on subsequent saccharin presentations (Clarke \& Westbrook, 1978; Riley, Hyson, Baker, \& Kulkosky, 1980; Riley, Lotter, \& Kulkosky, 1979; Roll, Schaeffer, \& Smith, 1969). This relative insensitivity of scheduleinduced polydipsia (SIP) to taste aversions is in marked contrast to the strong and long-lasting effects conditioned aversions have on water-deprivation-induced or postprandial drinking (Riley et al., 1980). It has been suggested that this relative insensitivity of SIP reflects a general tendency to drink and sample the conditioned stimulus following the spaced presentations of food (Riley et al., 1979). This increased tendency to drink is sufficient to override a conditioned aversion to the previously poisoned solution, as in other procedures that greatly elevate the likelihood of consumption and sampling, for example, extreme deprivation or dipsogenic agents (Grote \& Brown, 1973; Riley \& Lovely, 1978).

In the present experiment, animals were given access to both a drinking tube and a running wheel during the daily sessions in which they received food pellets on a variable-time 60 -sec schedule. Earlier re-

Requests for reprints should be sent to Anthony L. Riley, Department of Psychology, The American University, Washington, D.C. 20016, or to Paul J. Kulkosky, Edward W. Bourne Behavioral Research Lab, New York Hospital, Cornell Medical Center, Westchester Division, 21 Bloomingdale Road, White Plains, New York 10605. ports have demonstrated that the availability of a second recordable behavior, for example, wheel running, lowers the level of schedule-induced drinking (Levitsky \& Collier, 1968; Segal, 1969; Staddon \& Ayres, 1975). Since concurrent availability of a drinking tube and running wheel should lower the probability of drinking and the repeated pellet-induced sampling of the previously poisoned solution, such an opportunity for alternative behaviors might reduce the apparent insensitivity of SIP to conditioned aversions (Riley et al., 1980).

\section{METHOD}

\section{Subjects}

Subjects were six experimentally naive, female rats of LongEvans descent, approximately 90 days of age at the beginning of the experiment. The subjects were maintained on a 12-h-light/ 12-h-dark cycle for the duration of the experiment.
Apparatus
The subjects were housed in individual wire-mesh cages. In the front of each cage were openings into which graduated Nalgene tubes were placed for presentations of plain tap water or saccharin in the tap-water vehicle $(.1 \% \mathrm{w} / \mathrm{v}$ sodium saccharin, Fisher puri- fied) during conditioned aversion training. A single experimental apparatus was used for the daily free-food sessions. The apparatus consisted of a running wheel, $160 \mathrm{~cm}$ in diameter and $9 \mathrm{~cm}$ wide, with an $11.5 \times 15.5 \times 5.5 \mathrm{~cm}$ chamber attached to its lower front. A food tray was centered on the door of this chamber, $3.5 \mathrm{~cm}$ above the floor, A No. 1829 24-V dc lamp located directly above the food tray served as the magazine light. The back wall of the running wheel was constructed of 1-mm-thick aluminum. The front wall of the wheel and the attached chamber were constructed of 3-mm-thick Plexiglas, while the floor of the wheel and of the 
chamber was made of 6-mm hardware cloth. A graduated Nalgene tube was located on the front wall of the attached chamber, $1 \mathrm{~cm}$ to the left of the food hopper and $3.5 \mathrm{~cm}$ above the floor. The spout of the drinking tube protruded approximately $1.5 \mathrm{~cm}$ into the chamber. A complete rotation of the wheel was recorded by the deflection of a microswitch. A force of $20 \mathrm{~g}$ applied in either direction was sufficient to initiate rotation of the wheel.

\section{Procedure}

Phase 1: Conditioned aversion training. On Day 1, all subjects were deprived of water and given ad-lib access to food. On the next day, the subjects were given 20 -min access to water in the home cage. This procedure was repeated for 8 consecutive days, at which point all subjects were approaching and drinking from the tube within 2 sec of its presentation. On Day 9, all subjects were given 20-min access to saccharin, followed $15 \mathrm{~min}$ later by an intraperitoneal (ip) injection of $1.8 \mathrm{mEq} / \mathrm{kg}, .15 \mathrm{M}$ lithium chloride. On the following day, all subjects had 20 -min access to water in a waterrecovery session. On Day 11 , the subjects were again given 20 -min access to saccharin, followed $15 \mathrm{~min}$ later by an ip injection of $\mathrm{LiCl}$.

Phase 2: Schedule-induced polydipsia acquisition. On the day following the second conditioning trial, all subjects were given ad-lib access to food and water. Two days later, the food was removed, and the subjects were reduced to $85 \%$ of ad-lib-feeding body weight. Following this reduction in weight, all subjects were placed in the experimental chamber for a daily 30-min feeding session.

On Day 1 of Phase 2, a single 45-mg Noyes pellet was delivered to each subject, independently of its behavior, on average every $60 \mathrm{sec}$ (variable-time 60 -sec schedule) until each subject had received a total of 30 food pellets. Water was continuously available to all subjects during the spaced feedings. The wheel was locked, thus restricting the opportunity to run during this phase. This procedure was continued for $\mathbf{2 1}$ consecutive days.

Water intakes were measured at the conclusion of the 30-min session. If necessary, supplemental feedings of Purina Rat Chow were given to subjects in the home cage $10 \mathrm{~min}$ after each daily session to maintain body weights at $85 \%$ of initial values.

Phase 3: Concurrent availability of schedule-induced polydipsia and wheel running. On Day 1 of Phase 3, all subjects received 30 45-mg food pellets in 30 spaced meals. As above, water was continuously available to the subjects during the VT 60 -sec, free-food deliveries. In addition to water availability, on Day 1, the wheel was unlocked, allowing the animals unrestricted opportunity to run. On the following day, the wheel was again locked so that the subjects had access to the drinking tube only during the free food presentations. The procedure of alternating wheel/tube and tube availability was continued for 24 days.

Phase 4: Conditioned taste aversions/schedule-induced polydipsia interaction. As in Phase 3, the procedure of alternating tube/wheel and tube availability continued throughout Phase 4. At this point, however, differential treatment was administered to two groups of subjects ( $n=3$ for each group) that were matched on suppression of saccharin consumption during the second conditioning trial in Phase 1.

On Day 1 of Phase 4, while both groups had concurrent access to the drinking tube and the wheel, the previously poisoned saccharin solution was substituted for water during the free food presentations for Group SO (saccharin-wheel open). Group SC (saccharin-wheel closed) had access to water in the drinking tube on this session. On the following day, the wheel was locked, and all subjects had access only to the drinking tube. For Group SC, the previously poisoned saccharin solution was substituted for water. Group SO had access to water in the tube during this session. Thus, Group SO had access to saccharin when the wheel was available and Group SC had access to saccharin when the wheel was closed.

This procedure, whereby Group SO had access to saccharin and the wheel and Group SC had access to saccharin alone, was continued until each group had received eight exposures to saccharin. No injections followed saccharin access during this extinction phase.

\section{RESULTS}

Determinations of statistical significance were made by $t$ tests for independent and for repeated observations with significance levels at $\mathrm{p}<.05$, two-tailed.

\section{Phase 1: Conditioned Aversion Training}

Over the 8 water-adaptation days, all subjects increased consumption of water, drinking approximately $12 \mathrm{ml} /$ day over the final 3 days of water adaptation. When saccharin replaced water on Day 9 , there was a slight, but nonsignificant, decrease in consumption from water baseline, each group drinking approximately $11 \mathrm{ml}$ of saccharin on this initial exposure.

All subjects decreased consumption of saccharin following the initial saccharin- $\mathrm{LiCl}$ pairing, drinking approximately $5 \mathrm{ml}$ on the second conditioning trial $[\mathrm{t}(5)=3.15]$.

\section{Phase 2: Schedule-Induced \\ Polydipsia Acquisition}

On Day 1 of water adaptation in Phase 2, the subjects drank approximately $1.5 \mathrm{ml}$ of water, and on Day 21 , they drank approximately $8 \mathrm{ml}$.

\section{Phase 3: Concurrent Availability of Schedule-Induced Polydipsia and Wheel Running}

On Day 1 of Phase 3, when subjects had access to both the drinking tube and the wheel, there was a significant decrease in water consumption below the mean amount consumed on the last 3 days of Phase 2 , during which time subjects had access only to the tube $[\mathrm{t}(5)=4.55]$. On Day 2, when the wheel was again locked and only the drinking tube was available, drinking increased to the water baseline displayed prior to wheel access, that is, Phase 2.

Figure 1 presents the amount of water consumed during the free-food presentations when the wheel

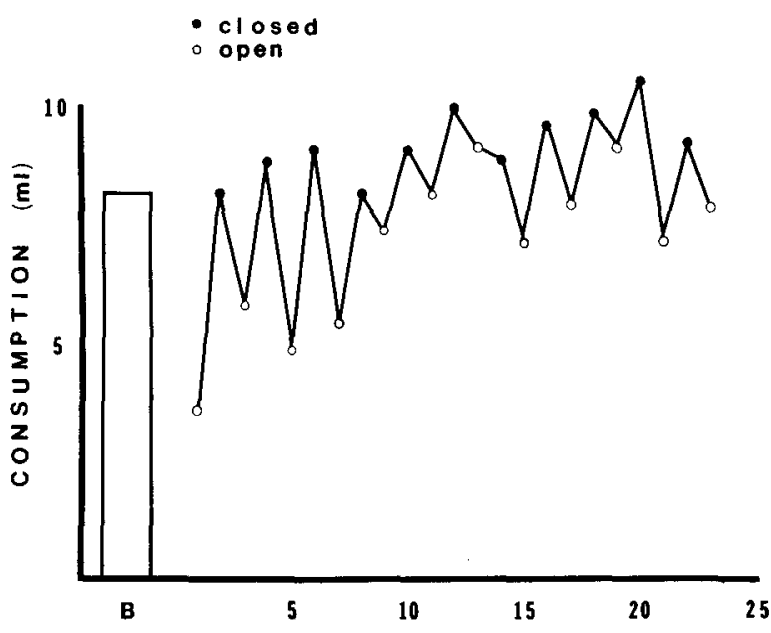

Figure 1. Mean consumption ( $\mathrm{ml}$ ) of water on the last 3 days of SIP baseline (B) of Phase 2 and during the free-food presentations when the wheel was open and closed. 
was open and closed. While the initial difference in water consumption between the wheel-open and wheel-closed conditions decreased with repeated sessions, on the last 2 days of this phase, five of the six subjects continued to drink more water when the wheel was closed than when the wheel was open.

\section{Phase 4: Conditioned Taste Aversions/ \\ Schedule-Induced Polydipsia Interaction}

Figure 2 presents the mean amount of water consumed in the wheel-closed and -open conditions for all subjects during the last 6 days of Phase 3 and the amount of saccharin consumed for Groups SC and SO over the eight saccharin presentations in Phase 4. While both Groups SC and SO avoided consumption of saccharin when saccharin was substituted for water on Day 1 of Phase 4 (means $=.50$ and $.49 \mathrm{ml}$; see Figure 2, Day 1), over subsequent extinction sessions, a difference between groups developed in the amount of saccharin consumed. Group SC, subjects given access to saccharin with the wheel locked, significantly increased saccharin consumption following the second nonpoisoned exposure to saccharin $[t(2)=$ 4.98]. With repeated exposures, this group continued to increase saccharin consumption, reaching asymptote by Day 5 . On the other hand, subjects in Group $\mathrm{SO}$, given access to both the previously poisoned saccharin solution and the wheel, showed no clear recovery of drinking until Day 5 , following four nonpoisoned exposures to saccharin. With repeated exposures to saccharin, this group further increased saccharin consumption, reaching asymptote by Day 7 . While Groups SC and SO did not differ in saccharin consumption on Day 1 of Phase 4, each drinking approximately $1 \mathrm{ml}$, Groups SC and SO drank approximately 17 and $7 \mathrm{ml}$, respectively, on the final extinction test (see Figure 2). Table 1 presents consump-

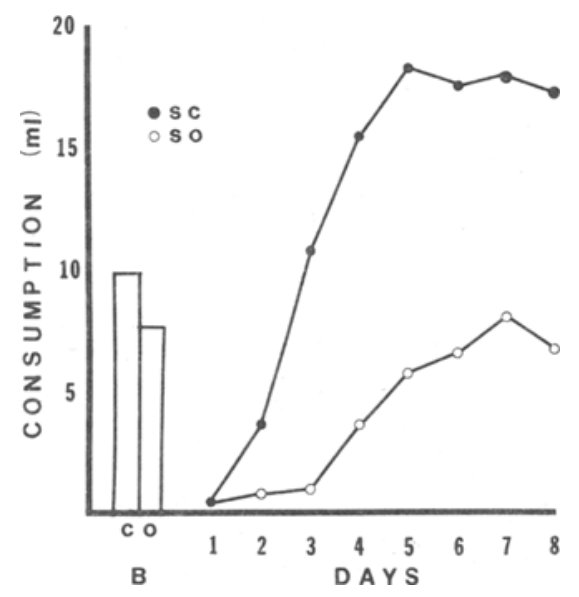

Figure 2. Mean consumption $(\mathrm{ml})$ of water in the wheel closed (C) and open (O) condition during the last 6 days of SIP baseline (B) of Phase 3 and of saccharin for Groups SC and SO during extinction.
Table 1

Consumption (in Milliliters) for Individual Subjects on the Last 3 Baseline (B) Days (When the Wheel Was Open for Group SO and Closed for Group SC) and Over the 8 Extinction Days

\begin{tabular}{rrrrrrr}
\hline & \multicolumn{7}{c}{ Consumption } \\
\cline { 6 - 8 } & \multicolumn{3}{c}{ Group SO } & \multicolumn{3}{c}{ Group SC } \\
\hline B & \multicolumn{1}{c}{ S2 } & S3 & S4 & S5 & \multicolumn{1}{c}{ S6 } \\
\hline 1 & 5.70 & 8.40 & 7.50 & 7.10 & 12.70 & 5.80 \\
2 & .00 & .00 & .00 & 1.20 & .30 & .00 \\
3 & 2.10 & .00 & .30 & 5.40 & 2.40 & 2.70 \\
4 & .90 & .00 & 1.20 & 16.50 & 6.60 & 9.30 \\
5 & 8.60 & .00 & 7.20 & 24.00 & 13.50 & 9.00 \\
6 & 11.70 & .00 & 8.40 & 23.10 & 21.00 & 11.40 \\
7 & 15.90 & .00 & 9.00 & 19.80 & 19.80 & 12.90 \\
8 & 11.70 & .00 & 8.40 & 20.10 & 21.00 & 12.90 \\
\hline
\end{tabular}

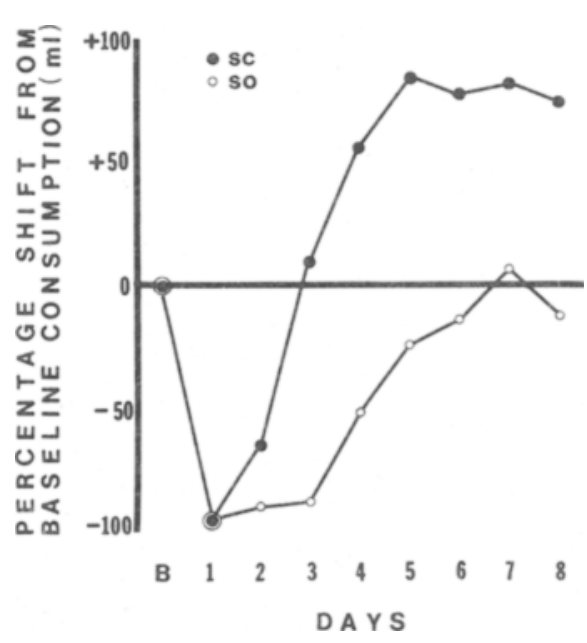

Figure 3. Percentage shift in consumption from the SIP baseline of Phase 3 to Phase 4. Phase 3 baselines for Group SO and Group SC were defined as the mean consumption over the last three sessions when the wheel was open (Group SO) and over the last three sessions when the wheel was closed (Group SC).

tion data for individual subjects in each group over Phase 4.

Because the baseline levels of water consumption under the wheel closed and open conditions differed, it is difficult to compare directly the recovery of saccharin consumption for Groups SC and SO. Figure 3 presents a transformation of the results illustrated in Figure 2 as respective percentages of baseline consumption (see Figure 2B). As can be seen in Figure 3, even when the fluid baselines are considered, consumption recovered more rapidly and completely for Group SC.

While the availability of running affected the recovery of schedule-induced drinking (see Figure 3), access to saccharin affected the level of wheel running. All three of the subjects given access to saccharin when the wheel was open (Group SO) showed wheel running above the level displayed when these subjects had access to water and the wheel during 


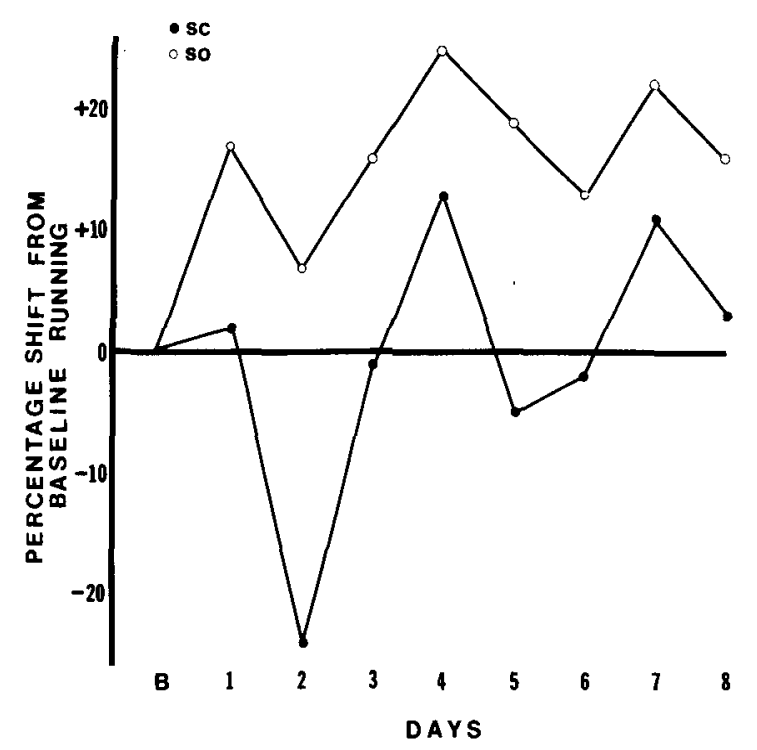

Figure 4. Percentage shift in running from Phase 3 baseline to Phase 4. For both groups, Phase 3 baselines were defined as the mean amount of running over the last three sessions when the wheel was open.

Phase 3. On the other hand, subjects given access to water when the wheel was open during Phase 4 (Group SC) did not show any consistent change in their level of running from the Phase 3 baseline. Figure 4 presents the percentage shift in running from Phase 3 to Phase 4 when subjects were given either saccharin (Group SO) or water (Group SC) and access to running. The tendency for the percentage shift in running to be greater for Group SO than for Group SC was significant by a binomial test $(p<.01)$.

\section{DISCUSSION}

It is clear from these data that the availability of wheel running prolonged the suppression of the schedule-induced drinking of a solution previously paired with poison. When running was restricted, the suppression of SIP was weak and transient, an insensitivity characteristic of schedule-induced drinking (Riley et al., 1980; Riley et al., 1979).

It has been suggested that the relative insensitivity of SIP to conditioned aversions reflects a general tendency to drink induced by the spaced pellet deliveries. As animals sample the aversive solution and are not subsequently poisoned, the aversion extinguishes, increasing the likelihood of further schedule-induced drinking. That the concurrent availability of a second behavior, for example, wheel running, both lowered the level of schedule-induced drinking in general (cf. Levitsky \& Collier, 1968; Segal, 1969; Phase 3) and retarded the recovery of schedule-induced drinking suppressed by taste aversions supports this interpretation.

As described above, access to wheel running lowered the overall level of schedule-induced drinking. As presented in Phase 4, the substitution of a previously poisoned solution for water during the free food presentations, while decreasing schedule-induced polydipsia, increased the overall level of wheel running. That access to running was associated with a decrease in drinking and that suppression of SIP by taste aversions was associated with an increase in running suggest that the level of either of these behaviors, that is, drinking or running, is in part interdependent upon the occurrence of the other. Such behavioral interdependency has previously been noted by Staddon and Ayres (1975). Given that wheel running is not typically described as being scheduleinduced (see Penny \& Schull, 1977; Staddon, 1977; Staddon \& Ayres, 1975) further suggests that the behavioral interdependency may not be restricted to schedule-induced behaviors.

\section{REFERENCES}

Clarke, J., \& Westbrook, R. Control of polydipsic drinking by a taste aversion procedure. Pharmacology, Biochemistry, \& Behavior, 1978, 9, 283-286.

Grote, F., \& Brown, R. Deprivation level affects extinction of a conditioned taste aversion. Learning and Motivation, 1973, 4, 314-319.

Levitsky, D., \& Collie r, G. Schedule-induced wheel running. Physiology \& Behavior, 1968, 3, 571-573.

Penny, J., \& Schuld, J. Functional differentiation of adjunctive drinking and wheel running in rats. Animal Learning \& Behavior, 1977, 5, 272-280.

Riley, A., Hyson, R., Baker, C., \& Kulkosky, P. The interaction of conditioned taste aversions and schedule-induced poly. dipsia: Effects of repeated conditioning trials. Animal Learning \& Behavior, 1980, 8, 211-217.

Riley, A., Lotter, E., \& Kulkosky, P. The effects of conditioned taste aversions on the acquisition and maintenance of schedule-induced polydipsia. Animal Learning \& Behavior, 1979, 7, 3-12.

RiLeY, A., \& Lovely, R. Chlordiazepoxide-induced reversal of an amphetamine-established aversion: Dipsogenic effects. Physiological Psychology, 1978, 6, 488-492.

Roll, D., Schaeffer, R., \& Smith, J. Effects of a conditioned taste aversion on schedule-induced polydipsia. Psychonomic Science, 1969, 16, 39-41.

SEgal, E. The interaction of psychogenic polydipsia with wheel running in rats. Psychonomic Science, 1969, 14, 141-142.

Staddon, J. Schedule-induced behavior. In W. Honig \& J. Staddon (Eds.), Handbook of operant behavior. Englewood Cliffs, N.J: Prentice-Hall, 1977.

Staddon, J., \& Ayres, S. Sequential and temporal properties of behavior induced by a schedule of periodic food delivery. Behavior, 1975, 54, 26-49.

(Received for publication September 16, 1980; revision accepted November 7,1980 .) 